\title{
Floquet spinor Bose gases
}

\author{
Kazuya Fujimoto $\oplus^{1}$ and Shun Uchino ${ }^{2}$ \\ ${ }^{1}$ Department of Physics, University of Tokyo, 7-3-1 Hongo, Bunkyo-ku, Tokyo 113-0033, Japan \\ ${ }^{2}$ Waseda Institute for Advanced Study, Waseda University, Shinjuku, Tokyo 169-8050, Japan
}

(Received 4 February 2019; published 26 November 2019)

\begin{abstract}
We introduce a Floquet spinor Bose-Einstein condensate induced by a periodically driven quadratic Zeeman coupling whose frequency is larger than any other energy scales. By examining a spin-1 system available in ultracold atomic gases, we demonstrate that such an external driving field has great effect on the condensate through emergence of a unique spin-exchange interaction. We uncover that the ferromagnetic condensate has several unconventional stationary states and thus exhibits rich continuous phase transitions. On the other hand, the antiferromagnetic condensate is found to possess a nontrivial metastable region, which supports unusual elementary excitations and hysteresis phenomena.
\end{abstract}

DOI: 10.1103/PhysRevResearch.1.033132

\section{INTRODUCTION}

Quantum degenerate systems with multiple order parameters emerge in diverse fields of physics such as unconventional superconductors $[1,2]$ and superfluid ${ }^{3} \mathrm{He}[3,4]$ in condensed matter, $p$-wave superfluids in neutron stars $[5,6]$, and color superconductors in quark matter $[7,8]$. Because of the presence of nontrivial order-parameter manifolds, such systems are known to exhibit a variety of phase structures, low-energy excitations, and topological defects absent in single order-parameter systems including conventional $s$-wave superconductors.

Currently, spinor Bose-Einstein condensates (BECs) realized in ultracold atomic gases offer testing grounds for examining fundamental properties of multiple order-parameter systems [9-14]. In fact, cold-atom experiments have successfully observed rich phase structures [15-17]; exotic topological excitations such as solitons [18-22], skyrmions [23-25], knots [26,27], and vortices [25,28,29]; and universal nonequilibrium dynamics [30-32].

One of the strengths in ultracold atomic gases is high controllability of experimental parameters, e.g., atom-photon interactions [33]. Of particular interest using this controllability is Floquet engineering [34-37], where a periodically oscillating field is applied to a system and thereby generates unconventional states absent in equilibrium $[38,39]$. In ultracold atomic gases, Floquet engineering has successfully been implemented [40-44], and one of the remarkable realizations is an artificial gauge field [45-48]. Despite the surge of great interest in Floquet engineering, such a technology in BECs has mainly been limited to engineering of kinetic energy terms.

Published by the American Physical Society under the terms of the Creative Commons Attribution 4.0 International license. Further distribution of this work must maintain attribution to the author(s) and the published article's title, journal citation, and DOI.
In this work, we introduce a Floquet spinor BEC induced by a high-frequency modulation of an external field (see Fig. 1) and uncover emergence of an unconventional spinexchange interaction. As a possible external field, we consider a microwave, which causes an effective quadratic Zeeman shift [16,17,49-52]. By employing high-frequency expansion in the Floquet formalism [46,53,54], we obtain an effective Hamiltonian having the unconventional interaction, which is in sharp contrast to the case of an artificial gauge field (note, however, Ref. [55]). Applying the theory of a weakly interacting Bose gas [56], we demonstrate that the effective Hamiltonian leads to several nontrivial stationary states and elementary excitations absent in the nondriven system. In the spinor BEC with a ferromagnetic (FM) interaction, we find emergence of the unconventional stationary states and various continuous phase transitions. The most notable state is a tilted broken axisymmetry (TBA) phase, which spontaneously breaks $Z_{2}$ symmetry despite absence of the linear Zeeman effect. In the system with an antiferromagnetic (AFM) interaction, on the other hand, we find a metastable region where two independent nonmagnetic states are stabilized simultaneously. This leads to hysteresis phenomena, which do not emerge in conventional spinor systems without the linear Zeeman effect [57].

This paper is organized as follows. In Sec. II, we introduce the theoretical model discussed in this work. In Sec. III, we apply the high-frequency expansion to the model. Section IV discusses possible stationary solutions for the obtained effective Hamiltonian. Sections V and VI respectively discuss metastable states and corresponding hysteresis phenomena. In Sec. VII, critical behaviors of the second-order transitions are analyzed. Section VIII discusses realizations of our proposal in cold-atom experiments. In Sec. IX, we summarize the paper. Some technical details on the Bogoliubov theory and critical behaviors are given in Appendixes.

\section{THEORETICAL MODEL}

We consider spin-1 atoms in a uniform system. Under the influence of a microwave, the Hamiltonian, $\hat{H}=\hat{H}_{\text {free }}+$ 


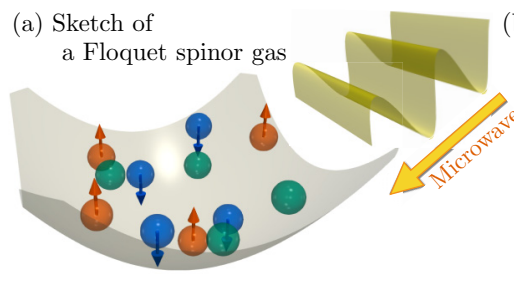

(b) Oscillation of a quadratic

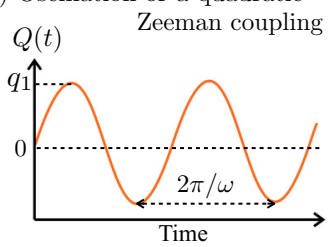

FIG. 1. (a) Schematic illustration of a Floquet spin-1 Bose gas induced by a microwave. Spheres with upward-pointing and downward-pointing arrows show particles with the magnetic sublevels $m=1$ and -1 , and those without arrows do particles with $m=0$. (b) A modulation of microwave's amplitude introduces a time-dependent quadratic Zeeman coupling $Q(t)$ with the frequency $\omega$ and the amplitude $q_{1}$.

$$
\begin{aligned}
\hat{H}_{\text {dint }}+\hat{H}_{\text {sint }}+\hat{H}_{\text {drive }}, \text { is given by }[10,11] & \\
\hat{H}_{\text {free }} & =\int d \boldsymbol{r} \sum_{m=-1}^{1} \hat{\psi}_{m}^{\dagger}(\boldsymbol{r})\left(-\frac{\hbar^{2}}{2 M} \nabla^{2}+q m^{2}\right) \hat{\psi}_{m}(\boldsymbol{r}), \\
\hat{H}_{\text {dint }} & =\frac{c_{0}}{2} \int d \boldsymbol{r}: \hat{n}(\boldsymbol{r}) \hat{n}(\boldsymbol{r}):, \\
\hat{H}_{\text {sint }} & =\frac{c_{2}}{2} \int d \boldsymbol{r}: \hat{\boldsymbol{F}}(\boldsymbol{r}) \cdot \hat{\boldsymbol{F}}(\boldsymbol{r}):, \\
\hat{H}_{\text {drive }} & =\int d \boldsymbol{r} \sum_{m=-1}^{1} Q(t) m^{2} \hat{\psi}_{m}^{\dagger}(\boldsymbol{r}) \hat{\psi}_{m}(\boldsymbol{r}) \\
: & =Q(t) \hat{D},
\end{aligned}
$$

where $\hat{\psi}_{m}(\boldsymbol{r})(m=1,0,-1)$ is the field operator of an atom with mass $M$ in a magnetic sublevel $m$ at position $r, c_{0}$ $\left(c_{2}\right)$ is the spin-independent (spin-dependent) coupling, $q$ is the static quadratic Zeeman coupling, $Q(t)$ is the driving Zeeman coupling, and :: denotes normal ordering. The density and spin density operators are respectively defined as $\hat{n}(\boldsymbol{r})=$ $\sum_{m=-1}^{1} \hat{\psi}_{m}^{\dagger}(\boldsymbol{r}) \hat{\psi}_{m}(\boldsymbol{r})$ and $\hat{F}_{\mu}(\boldsymbol{r})=\sum_{m, n=-1}^{1} \hat{\psi}_{m}^{\dagger}(\boldsymbol{r})\left(S_{\mu}\right)_{m n} \hat{\psi}_{n}(\boldsymbol{r})$ with the spin-1 matrix $\left(S_{\mu}\right)_{m n}(\mu=x, y, z)$.

\section{APPLICATION OF HIGH-FREQUENCY EXPANSION}

We focus on the case of the periodic driving $Q(t)=$ $q_{1} \cos (\omega t)$ with the amplitude $q_{1}$ and the frequency $\omega$ larger than any other timescales. Such an implementation is experimentally achievable via tuning a microwave [62]. By using high-frequency expansion [46,53,54], the system is well described by the following effective (static) Hamiltonian,

$$
\hat{H}_{\text {eff }}=\hat{H}_{0}+\frac{q_{1}^{2}}{4 \hbar^{2} \omega^{2}}\left[\left[\hat{D}, \hat{H}_{0}\right], \hat{D}\right]+\mathcal{O}\left(\omega^{3}\right)
$$

with $\hat{H}_{0}=\hat{H}_{\text {free }}+\hat{H}_{\text {dint }}+\hat{H}_{\text {sint }}$. Substituting Eqs. (1)-(5) into Eq. (6), we find that the double commutator gives an unconventional spin-dependent interaction as described in the following.

Let us start with noting that the operators $\hat{H}_{\text {free }}, \hat{H}_{\text {dint }}$, and $\hat{D}$ are invariant under the $U(1)$-gauge transformation $\hat{\psi}_{m} \rightarrow \hat{\psi}_{m} \exp \left(i \theta_{m}\right)$ with the phase factor $\theta_{m}$. This means that these operators conserve the particle number $\hat{N}_{m}$ for each component $m$ defined by

$$
\hat{N}_{m}=\int d \boldsymbol{r} \hat{\psi}_{m}^{\dagger}(\boldsymbol{r}) \hat{\psi}_{m}(\boldsymbol{r})
$$

This observation is related to the fact that the operator $\hat{D}$ commutes with $\hat{H}_{\text {free }}$ and $\hat{H}_{\text {dint }}$. In fact, the straightforward calculation leads to

$$
\left[\hat{D}, \hat{H}_{\text {free }}\right]=\left[\hat{D}, \hat{H}_{\text {dint }}\right]=0 .
$$

On the other hand, the spin-dependent interaction Hamiltonian $\hat{H}_{\text {sint }}$ does not commute with $\hat{D}$. This can be seen when we rewrite $\hat{H}_{\text {sint }}$ as follows:

$$
\hat{H}_{\text {sint }}=\frac{c_{2}}{2} \int d \boldsymbol{r}\left(: \hat{n}(\boldsymbol{r}) \hat{n}(\boldsymbol{r}):-3 \hat{A}^{\dagger}(\boldsymbol{r}) \hat{A}(\boldsymbol{r})\right)
$$

with the spin-singlet-pair operator $\hat{A}(\boldsymbol{r})$ defined by

$$
\hat{A}(\boldsymbol{r})=\frac{1}{\sqrt{3}}\left(2 \hat{\psi}_{1}(\boldsymbol{r}) \hat{\psi}_{-1}(\boldsymbol{r})-\hat{\psi}_{0}(\boldsymbol{r}) \hat{\psi}_{0}(\boldsymbol{r})\right) .
$$

The operator $\hat{A}^{\dagger}(\boldsymbol{r}) \hat{A}(\boldsymbol{r})$ clearly breaks the particle number conservation for each component and enhances the particle exchange between different spin components, which leads to the noncommutability between $\hat{D}$ and $\hat{H}_{\text {sint }}$. Using all the above results, we obtain

$$
\begin{aligned}
{\left[\hat{D}, \hat{H}_{0}\right]=} & -\frac{3 c_{2}}{2} \int d \boldsymbol{r}\left[\hat{D}, \hat{A}^{\dagger}(\boldsymbol{r}) \hat{A}(\boldsymbol{r})\right] \\
= & -2 c_{2} \int d \boldsymbol{r}\left(\hat{\psi}_{0}^{\dagger}(\boldsymbol{r}) \hat{\psi}_{0}^{\dagger}(\boldsymbol{r}) \hat{\psi}_{1}(\boldsymbol{r}) \hat{\psi}_{-1}(\boldsymbol{r})\right. \\
& \left.-\hat{\psi}_{0}(\boldsymbol{r}) \hat{\psi}_{0}(\boldsymbol{r}) \hat{\psi}_{1}^{\dagger}(\boldsymbol{r}) \hat{\psi}_{-1}^{\dagger}(\boldsymbol{r})\right) .
\end{aligned}
$$

In a similar manner, we can calculate the double commutator in Eq. (6):

$$
\begin{aligned}
{\left[\left[\hat{D}, \hat{H}_{0}\right], \hat{D}\right]=} & -4 c_{2} \int d \boldsymbol{r}\left(\hat{\psi}_{0}^{\dagger}(\boldsymbol{r}) \hat{\psi}_{0}^{\dagger}(\boldsymbol{r}) \hat{\psi}_{1}(\boldsymbol{r}) \hat{\psi}_{-1}(\boldsymbol{r})\right. \\
& \left.+\hat{\psi}_{0}(\boldsymbol{r}) \hat{\psi}_{0}(\boldsymbol{r}) \hat{\psi}_{1}^{\dagger}(\boldsymbol{r}) \hat{\psi}_{-1}^{\dagger}(\boldsymbol{r})\right) .
\end{aligned}
$$

Therefore, substituting Eq. (12) into Eq. (6), we obtain the effective static Hamiltonian:

$\hat{H}_{\mathrm{eff}}=\hat{H}_{0}-c_{f} \int d \boldsymbol{r}\left(\hat{\psi}_{1}^{\dagger}(\boldsymbol{r}) \hat{\psi}_{-1}^{\dagger}(\boldsymbol{r}) \hat{\psi}_{0}(\boldsymbol{r}) \hat{\psi}_{0}(\boldsymbol{r})+\right.$ H.c. $)$

with $c_{f}=q_{1}^{2} c_{2} / \hbar^{2} \omega^{2}$. Thus, the fast driving of the quadratic Zeeman term induces the novel spin-exchange interaction originating from the commutation relation $\left[\hat{H}_{\text {drive }}, \hat{H}_{\text {sint }}\right]$. We note that symmetry of the effective Hamiltonian is same as that of $\hat{H}$, which is invariant under $\mathrm{U}(1)$ phase rotation, $\mathrm{U}(1)$ spin rotation along the $z$ direction, and $Z_{2}$ spin rotation along the transverse direction.

\section{STATIONARY SOLUTIONS}

We examine stationary states of Eq. (13) within the meanfield approximation where $\hat{\psi}_{m}$ are replaced by c-numbers $\Psi_{m}$. 
The mean-field energy functional is given by

$$
\begin{aligned}
E\left[\Psi_{m}, \Psi_{m}^{*}\right]= & \int d \boldsymbol{r} \sum_{m=-1}^{1} \Psi_{m}^{*}(\boldsymbol{r})\left(-\frac{\hbar^{2}}{2 M} \nabla^{2}+q m^{2}\right) \Psi_{m}(\boldsymbol{r}) \\
& +\int d \boldsymbol{r}\left(\frac{c_{0}}{2} n(\boldsymbol{r})^{2}+\frac{c_{2}}{2} \boldsymbol{F}(\boldsymbol{r})^{2}\right) \\
& -c_{f} \int d \boldsymbol{r}\left(\Psi_{1}^{*}(\boldsymbol{r}) \Psi_{-1}^{*}(\boldsymbol{r}) \Psi_{0}(\boldsymbol{r}) \Psi_{0}(\boldsymbol{r})+\text { c.c. }\right),
\end{aligned}
$$

where the particle number density and the spin density vector are defined by $n=\sum_{m=-1}^{1}\left|\Psi_{m}\right|^{2}$ and $F_{\mu}=$ $\sum_{m, n=-1}^{1} \Psi_{m}^{*}\left(S_{\mu}\right)_{m n} \Psi_{n}$, respectively. In what follows, we focus on uniform solutions of Eq. (14) and the macroscopic wave function $\Psi_{m}$ is assumed to be independent of space. Then, the functional derivative with respective of $\Psi_{m}$ leads to the following coupled Gross-Pitaevskii equation:

$$
\begin{aligned}
& \mu \Psi_{1}=\left(q+c_{2} F_{z}\right) \Psi_{1}+\frac{c_{2}}{\sqrt{2}} F_{-} \Psi_{0}-c_{f} \Psi_{0}^{2} \Psi_{-1}^{*}, \\
& \mu \Psi_{0}=\frac{c_{2}}{\sqrt{2}}\left(F_{+} \Psi_{1}+F_{-} \Psi_{-1}\right)-2 c_{f} \Psi_{1} \Psi_{0}^{*} \Psi_{-1}, \\
& \mu \Psi_{-1}=\left(q-c_{2} F_{z}\right) \Psi_{-1}+\frac{c_{2}}{\sqrt{2}} F_{+} \Psi_{0}-c_{f} \Psi_{0}^{2} \Psi_{1}^{*}
\end{aligned}
$$

with $F_{ \pm}=F_{x} \pm i F_{y}$ and the chemical potential $\mu$ including the spin-independent interaction $c_{0} n_{0}$ with a uniform density $n_{0}$.

Denoting the macroscopic wave function by $\boldsymbol{\Psi}=\sqrt{n_{0}} \boldsymbol{\eta}$ with a vector $\boldsymbol{\eta}$ and analytically solving Eqs. (15)-(17), we find the following six stationary states. As known (inert) states, we obtain an FM state $\eta_{\mathrm{FM}}=(1,0,0)$, a polar state $\eta_{\mathrm{P}}=(0,1,0)$, and an AFM state $\eta_{\mathrm{AFM}}=(1,0,1) / \sqrt{2}[10,11]$. The rest are represented as

$$
\begin{gathered}
\eta_{\mathrm{BA}}=\left(\alpha, \sqrt{1-2 \alpha^{2}}, \alpha\right), \\
\eta_{\mathrm{TBA}}=\left(\alpha-\beta / 2, \sqrt{1-2 \alpha^{2}-\beta^{2} / 2}, \alpha+\beta / 2\right), \\
\eta_{\mathrm{TP}}=\left(-\beta / 2, \sqrt{1-\beta^{2} / 2}, \beta / 2\right)
\end{gathered}
$$

with $\alpha=\sqrt{\left(c_{f} n_{0}-2 c_{2} n_{0}-q\right) /\left(4 c_{f} n_{0}-8 c_{2} n_{0}\right)}$ and $\beta=$ $\sqrt{1+q / c_{f} n_{0}}$. The state $\eta_{\mathrm{BA}}$ represents a broken-axisymmetry (BA) state [63], where the nonzero magnetization lies in the $x-y$ plane as shown in Fig. 2(a), and U(1) phase and spin symmetries are spontaneously broken and the remaining symmetry is $Z_{2}$. Note that the energy is preserved regardless of rotations around the $z$ axis.

The tilted broken axisymmetry (TBA) state $\eta_{\mathrm{TBA}}$ and tilted polar (TP) one $\eta_{\mathrm{TP}}$ are unique to the Floquet spinor BEC, and the spin configurations and the Majorana representations are shown in Figs. 2(b) and 2(c). The TBA state $\eta_{\mathrm{TBA}}$ has the negative value of $S_{z}$ and can point to any directions in the $x-y$ plane in the same way as the BA state. Because of symmetry of Eq. (13) about interchange between $\Psi_{1}$ and $\Psi_{-1}$, $S_{z}$ can be positive, which means that $Z_{2}$ symmetry of the effective Hamiltonian is spontaneously broken in the TBA state. Therefore, the TBA state should be distinguished from the similar state in the ferromagnetic spinor BEC with the linear Zeeman term where $Z_{2}$ symmetry along the $z$ axis is absent at the Hamiltonian level. On the other hand, $\eta_{\mathrm{TP}}$ has
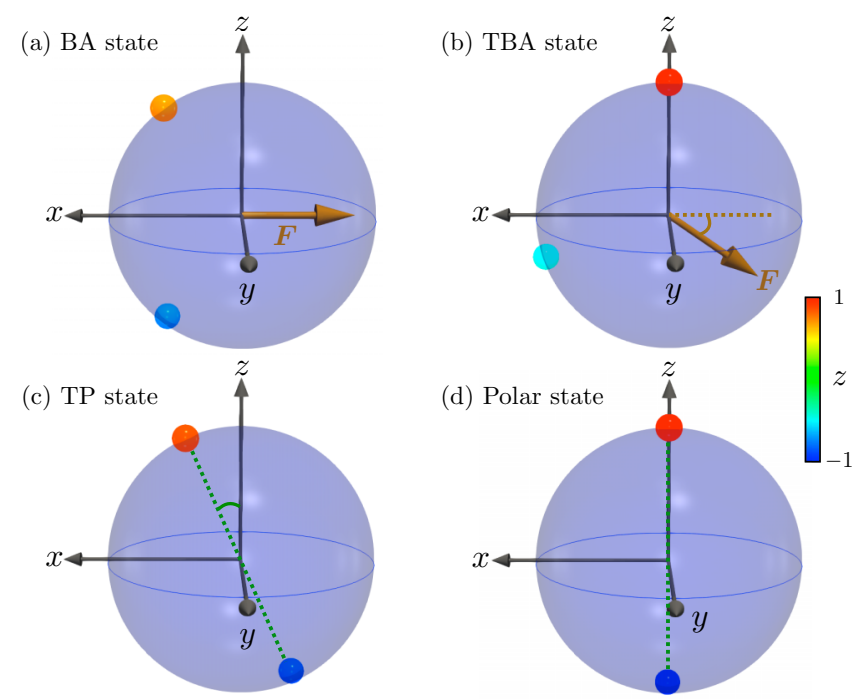

FIG. 2. Magnetization $\boldsymbol{F}$ and Majorana representation for (a) BA, (b) TBA, (c) TP, and (d) polar states. The magnetization in the BA state is restricted in the $x-y$ plane, while that in the TBA state has the nonzero $z$ component. In contrast, the TP and the polar states have no magnetization. Symmetry of each phase can geometrically be visualized with the Majorana representation expressed by two spheres $[10,11,67]$. Colors show values of $z$ for each sphere. If spheres are invariant under a transformation, a state has the corresponding symmetry.

zero magnetization, and the Majorana representation of $\boldsymbol{\eta}_{\mathrm{TP}}$ is seen to be tilted in compared with $\eta_{\mathrm{P}}$ as shown in Figs. 2(c) and 2(d). Thus, we name it a TP state.

We can obtain phase diagrams by evaluating energies of these stationary states. Figure 3(a) is the result for the FM interaction case $\left(c_{2}<0\right)$, which contains the nontrivial states, $\boldsymbol{\eta}_{\mathrm{TBA}}$ and $\boldsymbol{\eta}_{\mathrm{TP}}$. We can discuss orders of the transitions between these different phases by calculating two derivatives of total energy $\partial E\left(q, c_{f}\right) / \partial c_{f}$ and $\partial E\left(q, c_{f}\right) / \partial q$. Figures 3(c), 3(d) and 3(e) show $\partial E\left(q, c_{f}\right) / \partial q$ in three cases. As a result, we find that for $-2<c_{f} /\left|c_{2}\right|<0$, all transitions become second order. This is in sharp contrast to the non-driving case $\left(c_{f}=0\right)$ where a second-order transition is achieved only at the boundary between the BA and the polar phases.

Figure 3(b) is the phase diagram for the AFM interaction case $\left(c_{2}>0\right)$, where the transition line between the AFM and polar phases is independent of $c_{f}$ and is first order as shown in Fig. 3(f). As discussed in the next section, nontrivial metastable states are induced due to the periodically driving field.

\section{METASTABLE STATES FOR $c_{2}>0$}

The AFM spinor gas is known not to have metastable uniform stationary states when the linear Zeeman coupling is absent. In this section, we unveil existence of the metastable states in the AFM Floquet spinor Bose gas. We first numerically solve Eqs. (15)-(17) using the imaginary time-step method, in which independent Gaussian noises are added to the initial macroscopic wave functions. Figure 4(a) shows the numerical results, in which nematic tensor $\left(N_{z z}=\left|\Psi_{1}\right|^{2}+\right.$ $\left|\Psi_{-1}\right|^{2}$ ) exhibits the noisy behavior. This indicates presence of metastable states. 
(a) Phases for FM spinor gases

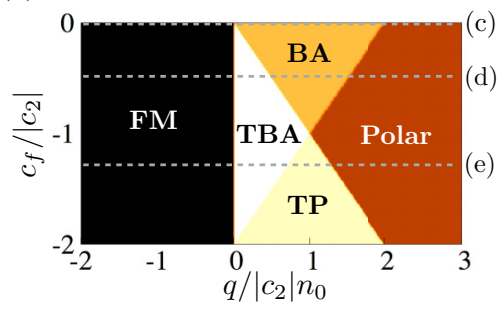

(b) Phases for AFM spinor gases

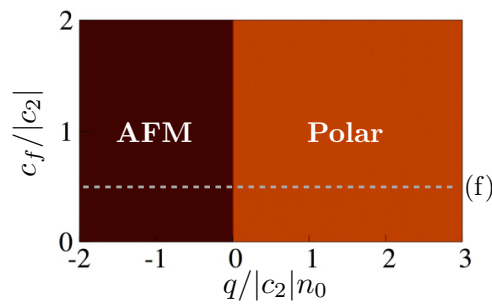

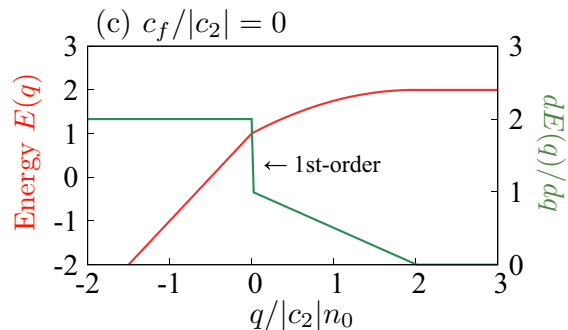
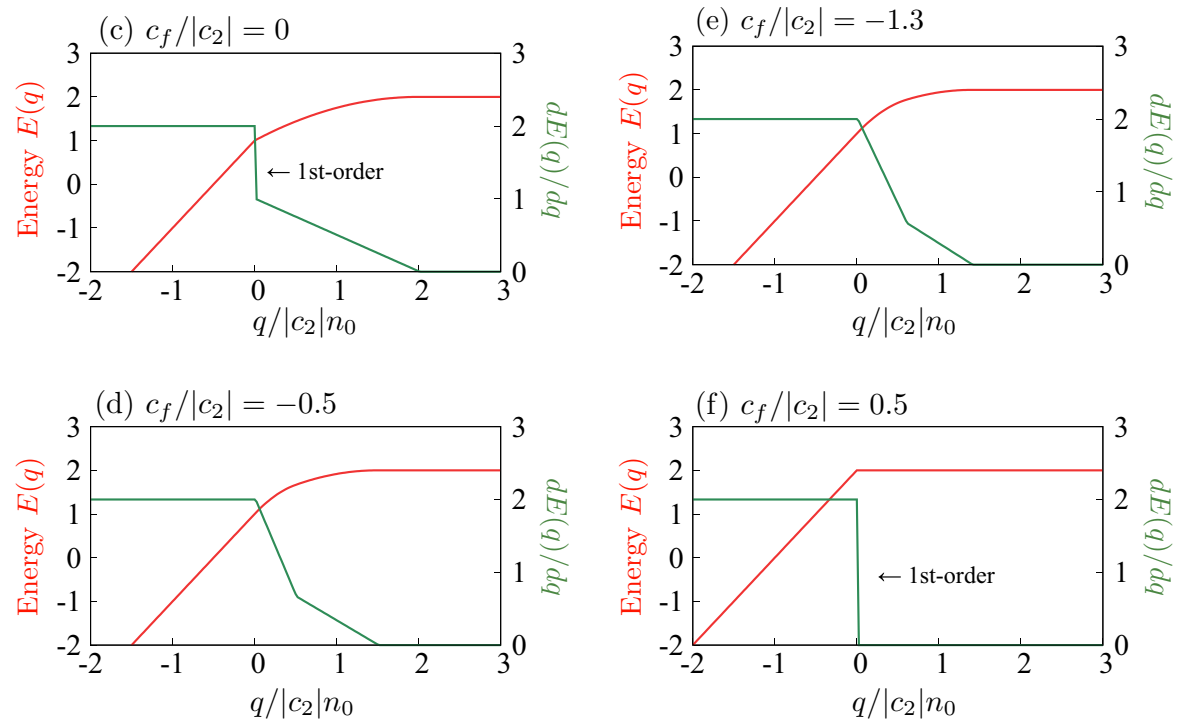

FIG. 3. (Left panels) Phase diagrams for (a) ferromagnetic and (b) antiferromagnetic interactions. In panel (a), while the FM, BA, and polar phases emerge in the nondriving case, the TBA and TP phases do only for nonzero $c_{f}$. In panel (b), the polar (AFM) state has the lowest energy when $q$ is positive (negative). (Middle and right panels) Dependences of mean-field energies and its derivative with respect to $q$. The units of ordinates are arbitrary units. The four panels show the dependences along (c), (d), (e), and (f) depicted in panels (a) and (b). Figures (c) and (f) clearly show the jumps of the derivative, which are hallmarks of a first-order phase transition.

We analytically understand the emergence of the metastable states for $c_{2}>0$ by looking at elementary excitations for $\eta_{\mathrm{P}}$ and $\eta_{\mathrm{AFM}}$. By employing the Bogoliubov theory [10,11] incorporating fluctuations of $\Psi_{m}$ up to second order, we obtain the Bogoliubov Hamiltonian in each phase from Eq. (13) and obtain elementary excitations known as the Bogoliubov modes (see Appendix A). In the AFM state, we have three excitations: a phonon mode related to U(1) phase symmetry, longitudinal spin mode, and transverse spin mode. These energy spectra are given by

$$
\begin{gathered}
E_{\mathrm{D}}^{(\mathrm{AFM})}(k)=\sqrt{\epsilon_{0, \boldsymbol{k}}\left(\epsilon_{0, \boldsymbol{k}}+2 c_{0} n_{0}\right)}, \\
E_{\mathrm{S}_{\mathrm{z}}}^{(\mathrm{AFM})}(k)=\sqrt{\epsilon_{0, \boldsymbol{k}}\left(\epsilon_{0, \boldsymbol{k}}+2 c_{2} n_{0}\right)}, \\
E_{\mathrm{S}_{\mathrm{xy}}}^{(\mathrm{AFM})}(k)= \begin{cases}-\sqrt{\left(\epsilon_{0, \boldsymbol{k}}-q+c_{2} n_{0}\right)^{2}-\left(c_{2} n_{0}-c_{f} n_{0}\right)^{2}} & \left(q / c_{2} n_{0}>1\right), \\
\sqrt{\left(\epsilon_{0, \boldsymbol{k}}-q+c_{2} n_{0}\right)^{2}-\left(c_{2} n_{0}-c_{f} n_{0}\right)^{2}} & \text { (otherwise), }\end{cases}
\end{gathered}
$$

(a)

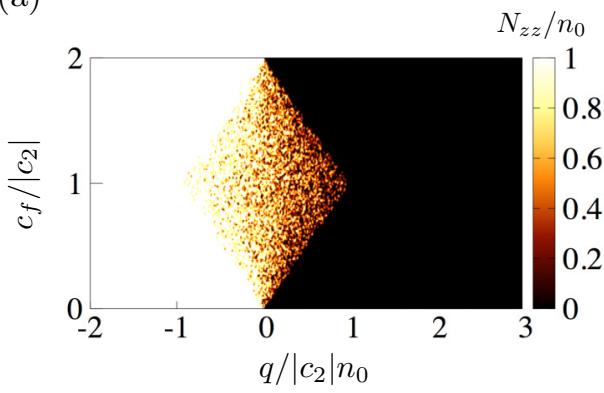

(b)

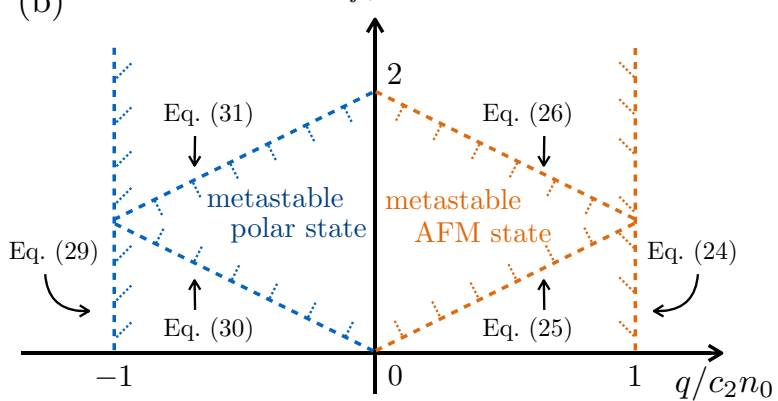

FIG. 4. (a) Parameter dependence of nematic tensor $N_{z z}=\left|\Psi_{1}\right|^{2}+\left|\Psi_{-1}\right|^{2}$ numerically obtained by the imaginary-time evolutions corresponding to Eqs. (15)-(17) with random initial noises. In the noisy region, $N_{z z} / n_{0}$ has unity or zero. This implies that the AFM (polar) state for positive (negative) $q$ can be metastable in the noisy region. (b) Metastable region in the AFM spinor Bose gas. The inequalities (24)-(26) and (29)-(31) determine the metastable regions. When $q$ is positive (negative), the AFM (polar) state becomes metastable. Outside of it, these metastable states become unstable against weak fluctuations and energy dissipation because the spectra have negative real or imaginary values. This is completely consistent with panel (a). 
where $\epsilon_{0, k}=\hbar^{2} k^{2} / 2 M$ is single-particle kinetic energy and $E_{\mathrm{D}}^{(\mathrm{AFM})}(k), E_{\mathrm{S}_{\mathrm{xy}}}^{(\mathrm{AFM})}(k)$, and $E_{\mathrm{S}_{\mathrm{z}}}^{(\mathrm{AFM})}(k)$ denote energy spectra for density, transverse spin, and longitudinal spin fluctuations, respectively. As usual, all the Bogoliubov modes take positive values for $q \leqslant 0$, where the AFM state is the lowest-energy state. What is surprising here is that these modes are positive even for the positive $q$ regime if the following inequalities are satisfied:

$$
\begin{gathered}
q<c_{2} n_{0}, \\
c_{f} n_{0}>q, \\
c_{f} n_{0}<-q+2 c_{2} n_{0} .
\end{gathered}
$$

Thus, the AFM state can be still stable for $q>0$, though it is not a lowest-energy state.

On the other hand, in the polar phase, we obtain a density mode and two degenerate spin modes whose energy spectra are given by

$$
E_{\mathrm{D}}^{(\mathrm{P})}(k)=\sqrt{\epsilon_{0, k}\left(\epsilon_{0, k}+2 c_{0} n_{0}\right)},
$$

$$
\begin{aligned}
& E_{\mathrm{S}}^{(\mathrm{P})}(k) \\
& \quad= \begin{cases}-\sqrt{\left(\epsilon_{0, \boldsymbol{k}}+q+c_{2} n_{0}\right)^{2}-\left(c_{2} n_{0}-c_{f} n_{0}\right)^{2}} & \left(q / c_{2} n_{0}<-1\right), \\
\sqrt{\left(\epsilon_{0, \boldsymbol{k}}+q+c_{2} n_{0}\right)^{2}-\left(c_{2} n_{0}-c_{f} n_{0}\right)^{2}} & \text { (otherwise). }\end{cases}
\end{aligned}
$$

As before, for $q \geqslant 0$, the polar state being the lowest-energy state is stable because the energy spectra (27) and (28) are positive. In addition, it can be stable even for a negative $q$ regime satisfying

$$
\begin{gathered}
q>-c_{2} n_{0}, \\
c_{f} n_{0}>-q, \\
c_{f} n_{0}<q+2 c_{2} n_{0} .
\end{gathered}
$$

The unusual stable region determined by Eqs. (24)-(26) and (29)-(31) means that the AFM and polar states can exist as metastable states, and the noisy region in Fig. 4(a) is indeed identical to it as shown in Fig. 4(b). Thus, we expect hysteresis loops when we adiabatically change $q, q_{1}$, or $\omega$ along a closed loop crossing the first-order transition.

We can discuss emergence of the metastable states from the perspective of symmetry of Eq. (13). In the absence of $c_{f}$, the transition between the AFM and the polar phases is known to be first order without a metastable state $[10,11]$, which is considered to be attributed to the restoration of $\mathrm{SO}(3)$ spin rotation symmetry at $q=0$ [64]. However, the nontrivial spin-exchange interaction proportional to $c_{f}$ breaks $\mathrm{SO}(3)$ rotational symmetry even at $q=0$, so that it can stabilize the polar (AFM) state in the limited negative (positive) regime.

\section{NUMERICAL INVESTIGATION FOR THE HYSTERESIS PHENOMENON}

As described in Sec. V, the Bogoliubov analysis predicts the hysteresis phenomenon in the AFM spinor Bose gas. In this section, we numerically demonstrate that slow sweep of the static quadratic Zeeman coupling $q$ can indeed induce the hysteresis curve.

\section{A. Formulation}

To consider the slow sweep of $q$ in the periodically driven spinor Bose gas, we use adiabatic Floquet theory constructed by Novičenko et al. [65]. Applying the formulation to the Hamiltonian (1)-(5) with the time-dependent $q(t)$, we can obtain the effective Hamiltonian:

$$
\begin{aligned}
\hat{H}_{\mathrm{eff}}= & \int d \boldsymbol{r} \sum_{m=-1}^{1} \hat{\psi}_{m}^{\dagger}(\boldsymbol{r})\left(-\frac{\hbar^{2}}{2 M} \nabla^{2}+q(t) m^{2}\right) \hat{\psi}_{m}(\boldsymbol{r}) \\
& +\frac{c_{0}}{2} \int d \boldsymbol{r}: \hat{n}(\boldsymbol{r}) \hat{n}(\boldsymbol{r}): \\
& +\frac{c_{2}}{2} \int d \boldsymbol{r}: \hat{\boldsymbol{F}}(\boldsymbol{r}) \cdot \hat{\boldsymbol{F}}(\boldsymbol{r}):-c_{f} \int d \boldsymbol{r}\left(\hat{\psi}_{1}^{\dagger}(\boldsymbol{r})\right. \\
& \left.\times \hat{\psi}_{-1}^{\dagger}(\boldsymbol{r}) \hat{\psi}_{0}(\boldsymbol{r}) \hat{\psi}_{0}(\boldsymbol{r})+\text { H.c. }\right) .
\end{aligned}
$$

This is almost same as Eq. (13) except for the time dependence of $q(t)$. We apply the mean-field approximation to Eq. (32) and obtain the equation of motion for the macroscopic wave functions $\Psi_{m}(m=1,0,-1)$ :

$$
\begin{aligned}
i \hbar \frac{\partial}{\partial t} \Psi_{1}= & -\frac{\hbar^{2}}{2 M} \nabla^{2} \Psi_{1}+q(t) \Psi_{1}+c_{0} n \Psi_{1} \\
& +c_{2}\left(\frac{1}{\sqrt{2}} F_{-} \Psi_{0}+F_{z} \Psi_{1}\right)-c_{f} \Psi_{-1}^{*} \Psi_{0}^{2}, \\
i \hbar \frac{\partial}{\partial t} \Psi_{0}=- & \frac{\hbar^{2}}{2 M} \nabla^{2} \Psi_{0}+c_{0} n \Psi_{0}+\frac{c_{2}}{\sqrt{2}}\left(F_{+} \Psi_{1}+F_{-} \Psi_{-1}\right) \\
- & 2 c_{f} \Psi_{1} \Psi_{0}^{*} \Psi_{-1}, \\
i \hbar \frac{\partial}{\partial t} \Psi_{-1}= & -\frac{\hbar^{2}}{2 M} \nabla^{2} \Psi_{-1}+q(t) \Psi_{-1}+c_{0} n \Psi_{-1} \\
& +c_{2}\left(\frac{1}{\sqrt{2}} F_{+} \Psi_{0}-F_{z} \Psi_{-1}\right)-c_{f} \Psi_{1}^{*} \Psi_{0}^{2} .
\end{aligned}
$$

We numerically solve Eqs. (33)-(35) starting from the polar state $\boldsymbol{\Psi}=\sqrt{n_{0}}(0,1,0)$ in a one-dimensional system with $c_{2}=c_{0} / 10$. In this simulation, we neglect a kick (micromotion) operator, which causes fast periodic oscillations of the observables with the frequency $\omega[34,65]$. This is because we expect that the effects may be suppressed by the ensemble average.

The control parameter $q(t)$ is assumed to obey

$$
q(t)=\left\{\begin{array}{ll}
q_{\mathrm{ini}}\left(1-\frac{2 t}{\tau_{\mathrm{ch}}}\right) & \left(0 \leqslant t \leqslant \tau_{\mathrm{ch}}\right) \\
q_{\mathrm{ini}}\left(-1+\frac{2 t-2 \tau_{\mathrm{ch}}}{\tau_{\mathrm{ch}}}\right) & \left(\tau_{\mathrm{ch}}<t \leqslant 2 \tau_{\mathrm{ch}}\right)
\end{array},\right.
$$

with the initial value $q_{\text {ini }}=0.4 c_{2} n_{0}$ and the characteristic time $\tau_{\mathrm{ch}}=10000 \hbar / c_{0} n_{0}$. The whole process for the parameter control is illustrated in Fig. 5.

To identify emergence of the hysteresis phenomenon, we need an order parameter for the AFM state. Then, we adopt a 


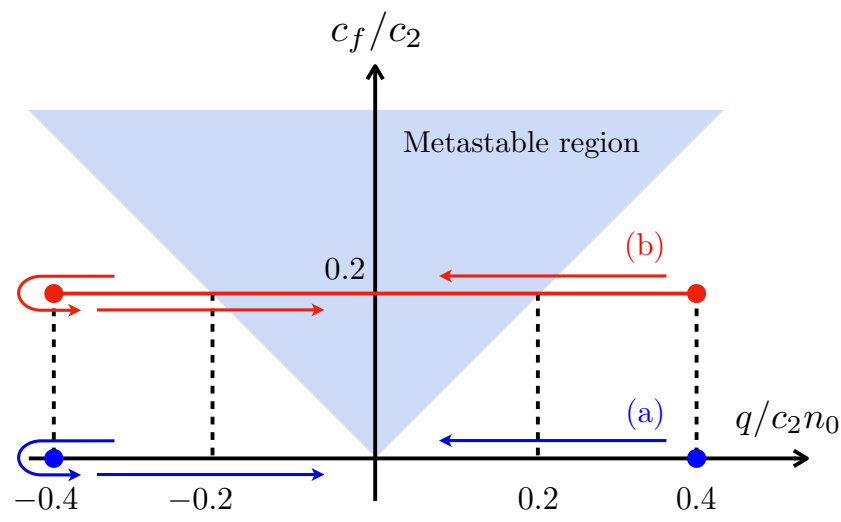

FIG. 5. Schematic for the metastable region and the paths of the control parameter $q(t)$. The shaded region denotes the metastable region where the AFM and polar states are not the lowest energy states in $q>0$ and $q<0$, respectively, but they are still metastable against weak fluctuations and energy dissipation. The numerical simulations consider two paths for (a) $c_{f} / c_{2}=0$ and (b) 0.2 where the parameter $q(t)$ obeys Eq. (36). The former path (a) does not cross the metastable region, and there is no hysteresis. On the other hand, the latter one (b) passes the region, and the hysteresis curve is expected to emerge.

nematic tensor for the $z z$ component defined by

$$
N_{z z}=\sum_{m, n=-1}^{1} \Psi_{m}^{*}\left(S_{z}^{2}\right)_{m n} \Psi_{n}=\left|\Psi_{1}\right|^{2}+\left|\Psi_{-1}\right|^{2} .
$$

The polar and the AFM states have $N_{z z}=0$ and $n_{0}$, respectively. Thus, by monitoring $N_{z z}$ in time, we can judge which states grow or decay in the dynamics.

\section{B. Numerical results}

We perform numerical simulations for (a) $c_{f}=0$ and (b) $c_{f}=0.2 c_{2}$ by starting from the polar state. As depicted in Fig. 5, the former protocol (a) does not pass the metastable region while the latter (b) crosses it. Thus, in the case (b), the initial polar state should remain stable just before the parameter gets across the point $q / c_{2} n_{0}=-0.2$ in the forward protocol. After getting out of the metastable region, the AFM order, namely $N_{z z}$, rapidly grows in time. Subsequently, $q$ turns at the point $q / c_{2} n_{0}=-0.4$, and the AFM state will survive even in the positive $q$ smaller than $0.2 c_{2} n_{0}$. In the following, we numerically test this scenario.

Figure 6 shows the time evolution of $N_{z z}$ for (a) $c_{f}=0$ and (b) $c_{f}=0.2 c_{2}$. In the nondriven case (a), the polar state in the negative $q$ region becomes unstable and the AFM state grows just after $q$ becomes negative. This can be seen in the rapid increase of $N_{z z}$. Here, note that the increase of $N_{z z}$ appears to occur around $q / c_{2} n_{0} \sim 0.03$ and there is a small time delay for the emergence of the instability. This is because the strength of the instability is small just after crossing the zero point and thus it takes a little more time for the growth of $N_{z z}$. In the backward path, the system shows the instability close to $q / c_{2} n_{0}=0$, and the polar state revives.

On the other hand, the driven case (b) exhibits quite different dynamics, as shown in Fig. 6(b). The polar state is still
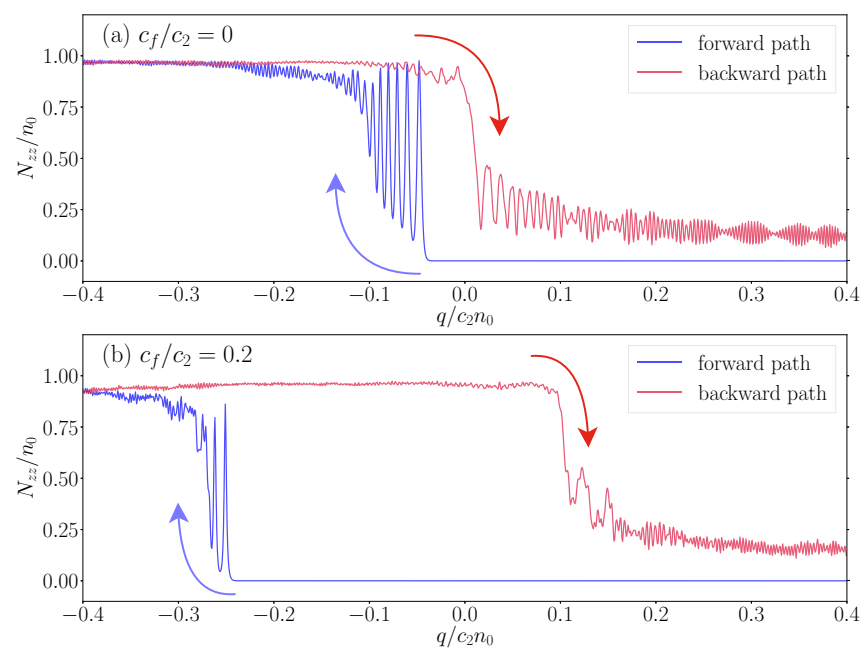

FIG. 6. Time evolution of $N_{z z}$ for the paths (a) and (b) in Fig. 5. The upper and lower panels show the numerical results of (a) and (b), respectively. Comparing (a) with (b), we confirm that the driven system shows a clear hysteresis curve (see text).

stable in the negative $q$ regime because of $N_{z z} \sim 0$. However, after the control parameter $q$ goes out of the metastable region, $N_{z z}$ grows in time. In the backward path, we also find that the AFM state survives even in the positive $q$ regime. Clearly, we find the hysteresis in the driven case, which is consistent with our theoretical prediction based on the Bogoliubov analysis. Here, we comment on the instability in the backward path of (b), where the instability point $q / c_{2} n_{0} \sim 0.1$ is a little different from our theoretical prediction. We expect that this is attributed to the energy change by the time evolution of $q(t)$. In the backward path, the continuous parameter change can make the condensates fluctuate and can enhance the instability. The similar dynamics is also seen in the nondriven case, where the instability in the backward path emerges faster than that in the forward path, as shown in Fig. 6(a). Finally, we comment on the experiment [66], in which the quadratic Zeeman coupling slowly oscillates in the AFM spinor Bose gas with ${ }^{23} \mathrm{Na}$, and the similar hysteresis phenomena are observed. However, the frequency is not so fast, and thus our theoretical prediction cannot readily be applicable to the experiment.

\section{CRITICAL BEHAVIORS FOR $c_{2}<0$}

We investigate critical behaviors near the second-order phase transitions in Fig. 3(a). In particular, we focus on (a) BA-polar, (b) BA-TBA, and (c) FM-TBA phase transitions, since these transitions emerge in $\left|c_{f} / c_{2}\right|<1$, where the effective Hamiltonian approach works well. For convenience, we introduce dimensionless variables $x=q /\left|c_{2}\right| n_{0}$ and $y=c_{f} /\left|c_{2}\right|$ and denote the parameter space as $\boldsymbol{R}=$ $(x, y)$. The details of the calculation shown here are given in Appendix B.

First, we consider the case (a), where the phase boundary is given by $\boldsymbol{R}_{0}=\left(x_{0}, x_{0}-2\right)\left(1<x_{0} \leqslant 2\right)$. As shown in in Fig. 7(a), a point around the phase boundary is specified as $\boldsymbol{R}=\left(x_{0}+r \cos \theta, x_{0}-2+r \sin \theta\right)$ with $r \ll 1$, where $r$ and $\theta$ are the polar coordinates. Then, longitudinal and transverse 


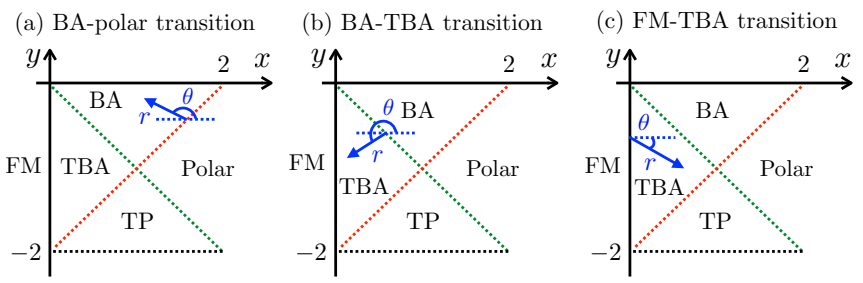

FIG. 7. Polar coordinates $(r, \theta)$ near the phase boundaries: (a) BA-polar, (b) BA-TBA, and (c) FM-TBA transitions.

magnetizations in the BA phase are expressed as

$$
F_{z}=0, \quad F_{\perp} \propto r^{1 / 2},
$$

while magnetizations in the polar phase vanish. As first pointed out in Ref. [68], such critical behaviors are same as those of the $X Y$ model.

In the case (b), the transition point is denoted as $\boldsymbol{R}_{0}=$ $\left(x_{0},-x_{0}\right)\left(0<x_{0}<1\right)$ as shown in Fig. 7(b). A point around the phase boundary is specified by $\boldsymbol{R}=\left(x_{0}+r \cos \theta,-x_{0}+\right.$ $r \sin \theta$ ) with $r \ll 1$. Then, longitudinal and transverse magnetizations in the TBA phase are given by

$$
F_{z} \propto r^{1 / 2}, \quad F_{\perp}-F_{0} \propto r
$$

with the transverse magnetization at $\boldsymbol{R}_{0}, F_{0}$. In terms of symmetry, what is essential here is that $Z_{2}$ spin rotational symmetry is spontaneously broken in the TBA phase while it is unbroken in the BA phase. Indeed, the leading variance on the magnetizations is the longitudinal one directly related to $Z_{2}$ spin rotational symmetry, and the transition type corresponds to the Ising model.

Finally, in the case (c), the transition point is denoted as $\boldsymbol{R}_{0}=\left(0, y_{0}\right)\left(-2<y_{0}<0\right)$. A point around the phase boundary is specified by $\boldsymbol{R}=\left(r \cos \theta, y_{0}+r \sin \theta\right)$ as shown in Fig. 7(c). The magnetization behaviors in the TBA phase are expressed as

$$
F_{z}-n_{0} \propto r, \quad F_{\perp} \propto r^{1 / 2},
$$

while in the FM phase the longitudinal magnetization coincides with $n_{0}$ and the transverse magnetization vanishes. In this transition, $\mathrm{U}(1)$ spin rotational symmetry is of importance since it is broken (unbroken) in the TBA (FM) phase. Therefore, the $X Y$-type transition is expected. In addition, by using the Bogoliubov theory, the dynamical critical exponent $z$ is obtained as $z=2$. Thus, the universality corresponds to that of a single-component dilute Bose gas [69].

\section{DISCUSSION}

We now discuss the validity of the high-frequency expansion. According to previous literature [70-73], when a discrete lattice system is locally bounded, $\hbar \omega$ must be larger than the local energy. While our system is spatially continuous, we may introduce the local chemical potential $\mu \sim c_{0} n_{0}$ as an effective cutoff. Thus, $\mu<\hbar \omega$ is a necessary condition for the validity. In addition, $\left|c_{f} / c_{2}\right|=\left|q_{1} / \hbar \omega\right|^{2} \ll 1$ is required to neglect higher order effects. Under these conditions, the fundamental properties in Floquet spinor BECs discussed above may be observed.

To realize an FM Floquet spin-1 BEC, we can consider ${ }^{87} \mathrm{Rb}$ and ${ }^{7} \mathrm{Li}$ as possible atomic species. Then, observation of the TBA state can be an experimental signature of the Floquet spinor BEC. The TBA state itself can be confirmed by emergence of a spin domain along the longitudinal direction, which is absent in the nondriving case for $q>0[10,11,74,75]$. On the other hand, it may be difficult to realize the TP phase in Fig. 3(a) because the condition $\left|c_{f} / c_{2}\right| \ll 1$ is broken. In addition, we can use ${ }^{23} \mathrm{Na}$ to realize an AFM Floquet spin-1 BEC. Then, the Floquet property is identified as presence of the metastable state, which can be measured through absorption images of magnetic sublevels in different times [58,61]. For example, we consider an experiment in which the polar state is initially prepared at $q<0$. In the absence of the driving, such a state is unstable against the dynamical instability and the $m= \pm 1$ components turn to grow up as a function of time. Namely, presence of metastability is proved as robustness of the $m=0$ component by the driving.

\section{CONCLUSION}

We have theoretically studied a spin-1 Bose gas under the periodically oscillating quadratic Zeeman coupling using the high-frequency expansion and the Bogoliubov theory. In the FM interaction case, we have found the emergence of the unconventional TBA and the TP states in addition to the known magnetic phases and the rich second-order phase transitions. On the other hand, the systems with the AFM interaction have the metastable states stabilized by the unusual Bogoliubov excitations, which can lead to hysteresis phenomena that do not emerge in undriven spinor BECs. We finally note that our method to engineer many-body interactions is applicable to various multicomponent quantum degenerate systems other than spinor Bose gases. For example, systems with strong dipole-dipole interactions, the Rabi coupling, etc. may give nontrivial interactions induced by periodically oscillating external fields.

\section{ACKNOWLEDGMENTS}

We would like to thank Y. Shin and M. Ueda for comments in early stage of this work, and also thank S. Furukawa, R. Hamazaki, S. Higashikawa, M. Nakagawa, and M. Sato for fruitful discussions. K.F. is supported by a JSPS fellowship (JSPS KAKENHI Grant No. JP16J01683). S.U. is supported by JSPS KAKENHI Grant No. JP17K14366 and a Waseda University Grant for Special Research Projects (No. 2019C-461).

\section{APPENDIX A: BOGOLIUBOV THEORY FOR THE EFFECTIVE HAMILTONIAN}

Applying the Bogoliubov theory [10] to the effective Hamiltonian (13), we derive energy spectra of elementary excitations for the polar and the AFM states. Let us start with rewriting the Hamiltonian (13) in terms of the bosonic annihilation and creation operators in the wavenumber space. The bosonic field operators are expanded as

$$
\begin{aligned}
& \hat{\psi}_{m}(\boldsymbol{r})=\frac{1}{\sqrt{V}} \sum_{\boldsymbol{k}} \hat{a}_{m, \boldsymbol{k}} e^{i \boldsymbol{k} \cdot \boldsymbol{r}}, \\
& \hat{\psi}^{\dagger}(\boldsymbol{r})=\frac{1}{\sqrt{V}} \sum_{\boldsymbol{k}} \hat{a}_{m, \boldsymbol{k}}^{\dagger} e^{-i \boldsymbol{k} \cdot \boldsymbol{r}},
\end{aligned}
$$


where $V$ denotes the system volume and the operators $\hat{a}_{m, k}$ and $\hat{a}_{m, k}^{\dagger}$ satisfy the bosonic commutation relations such as $\left[\hat{a}_{n, \boldsymbol{k}_{1}}, \hat{a}_{m, \boldsymbol{k}_{2}}^{\dagger}\right]=\delta_{\boldsymbol{k}_{1} \boldsymbol{k}_{2}} \delta_{n m}$. Substituting Eqs. (A1) and (A2) into (13), we obtain

$$
\begin{aligned}
\hat{H}_{\mathrm{eff}}= & \sum_{\boldsymbol{k}} \sum_{m} \epsilon_{m, \boldsymbol{k}} \hat{a}_{m, \boldsymbol{k}}^{\dagger} \hat{a}_{m, \boldsymbol{k}}+\frac{1}{V} \sum_{\boldsymbol{k}_{1}, \boldsymbol{k}_{2}, \boldsymbol{k}_{3}, \boldsymbol{k}_{4}} \sum_{m, n, p, q} \Gamma_{p q}^{m n} \delta\left(\boldsymbol{k}_{1}\right. \\
& \left.+\boldsymbol{k}_{2}-\boldsymbol{k}_{3}-\boldsymbol{k}_{4}\right) \hat{a}_{m, \boldsymbol{k}_{1}}^{\dagger} \hat{a}_{p, \boldsymbol{k}_{2}}^{\dagger} \hat{a}_{n, \boldsymbol{k}_{3}} \hat{a}_{q, \boldsymbol{k}_{4}} \\
& -\frac{c_{f}}{V} \sum_{\boldsymbol{k}_{1}, \boldsymbol{k}_{2}, \boldsymbol{k}_{3}, \boldsymbol{k}_{4}} \delta\left(\boldsymbol{k}_{1}+\boldsymbol{k}_{2}-\boldsymbol{k}_{3}-\boldsymbol{k}_{4}\right) \\
& \times\left(\hat{a}_{1, \boldsymbol{k}_{1}}^{\dagger} \hat{a}_{-1, \boldsymbol{k}_{2}}^{\dagger} \hat{a}_{0, \boldsymbol{k}_{3}} \hat{a}_{0, \boldsymbol{k}_{4}}+\text { H.c }\right)
\end{aligned}
$$

with the single-particle energy $\epsilon_{m, k}=\hbar^{2} k^{2} / 2 M+q m^{2}$ and the interaction coupling $\Gamma_{p q}^{m n}$ defined by

$$
\Gamma_{p q}^{m n}=\frac{c_{0}}{2} \delta_{n p} \delta_{m q}+\frac{c_{2}}{2}(\boldsymbol{S})_{m n} \cdot(\boldsymbol{S})_{p q} .
$$

We use the Bogoliubov approximation, in which the zero momentum operators $\hat{a}_{m, 0}(m=1,0,-1)$ are replaced by c-numbers $\phi_{m}=\sqrt{N_{0}} \eta_{m}(m=1,0,-1)$ with the condensed particle number $N_{0}$. By using the number conserving theory [10], the Hamiltonian (A4) is approximated to

$$
\begin{gathered}
\hat{H}_{\text {eff }} \simeq \hat{H}_{\text {kin }}+\hat{H}_{\text {int }}+\hat{H}_{\text {flo }}, \\
\hat{H}_{\text {kin }}=E_{\text {kin }}+\sum_{\boldsymbol{k} \neq \mathbf{0}} \sum_{m}\left\{\epsilon_{m, \boldsymbol{k}}-\sum_{n} \epsilon_{n, 0}\left|\eta_{n}\right|^{2}\right\} \hat{a}_{m, \boldsymbol{k}}^{\dagger} \hat{a}_{m, \boldsymbol{k}},
\end{gathered}
$$

$$
\begin{aligned}
\hat{H}_{\mathrm{int}}= & E_{\mathrm{int}}-n_{0}\left(c_{0}+c_{2} \boldsymbol{f}^{2}\right) \sum_{m} \sum_{\boldsymbol{k} \neq \mathbf{0}} \hat{a}_{m, \boldsymbol{k}}^{\dagger} \hat{a}_{m, \boldsymbol{k}} \\
& +n_{0} \sum_{m, n, p, q} \sum_{\boldsymbol{k} \neq \mathbf{0}}\left(\Gamma_{p q}^{m n} \eta_{m}^{*} \eta_{p}^{*} \hat{a}_{n, \boldsymbol{k}} \hat{a}_{q,-\boldsymbol{k}}+\Gamma_{p q}^{m n} \eta_{n} \eta_{q} \hat{a}_{m, \boldsymbol{k}}^{\dagger} \hat{a}_{p,-\boldsymbol{k}}^{\dagger}\right) \\
& +n_{0} \sum_{m, n, p, q} \sum_{\boldsymbol{k} \neq \mathbf{0}}\left(\Gamma_{p q}^{m n}+\Gamma_{m n}^{p q}+\Gamma_{m q}^{p n}+\Gamma_{p n}^{m q}\right) \eta_{p}^{*} \eta_{q} \hat{a}_{m, \boldsymbol{k}}^{\dagger} \hat{a}_{n, \boldsymbol{k}}, \\
\hat{H}_{\text {flo }}= & E_{\mathrm{flo}}+2 c_{f} n_{0} \eta_{1}^{*} \eta_{-1}^{*} \eta_{0}^{2} \sum_{\boldsymbol{k} \neq \mathbf{0}} \sum_{m} \hat{a}_{m, \boldsymbol{k}}^{\dagger} \hat{a}_{m, \boldsymbol{k}} \\
& -c_{f} n_{0} \sum_{\boldsymbol{k} \neq \mathbf{0}}\left(\eta_{1}^{*} \eta_{-1}^{*} \hat{a}_{0, \boldsymbol{k}} \hat{a}_{0,-\boldsymbol{k}}+2 \eta_{1}^{*} \eta_{0} \hat{a}_{-1, \boldsymbol{k}}^{\dagger} \hat{a}_{0, \boldsymbol{k}}\right. \\
& \left.+2 \eta_{-1}^{*} \eta_{0} \hat{a}_{1, \boldsymbol{k}}^{\dagger} \hat{a}_{0, \boldsymbol{k}}+\eta_{0}^{2} \hat{a}_{1, \boldsymbol{k}}^{\dagger} \hat{a}_{-1,-\boldsymbol{k}}^{\dagger}\right)+ \text { H.c. }
\end{aligned}
$$

with the total particle number density $n_{0}=N / V$, the total particle number $N$, the normalized spin vector $f_{\mu}=$ $\sum_{m, n=-1}^{1} \eta_{m}^{*}\left(S_{\mu}\right)_{m n} \eta_{n}$, and the trivial constants $E_{\text {kin }}, E_{\text {int }}$, and $E_{\text {flo. }}$. The quadratic Hamiltonian (A5)-(A8) is the general form for any stationary states. In what follows, we consider the polar and the AFM states, and analytically calculate the energy spectra.

\section{Polar state}

We derive the energy spectra of elementary excitations in the polar state using Eqs. (A5)-(A8). The spinor wave function is given by $\eta_{\mathrm{P}}=(0,1,0)$, which leads to the following Bogoliubov Hamiltonian:

$$
\begin{gathered}
\hat{H}_{\text {polar }}=\hat{H}_{\text {polar }}^{(1)}+\hat{H}_{\text {polar }}^{(2)}+\text { const., } \\
\hat{H}_{\text {polar }}^{(1)}=\frac{1}{2} \sum_{\boldsymbol{k} \neq \mathbf{0}}\left(\hat{a}_{0, \boldsymbol{k}}^{\dagger}, \hat{a}_{0,-\boldsymbol{k}}\right)\left(\begin{array}{cc}
\epsilon_{0, \boldsymbol{k}}+c_{0} n_{0} & c_{0} n_{0} \\
c_{0} n_{0} & \epsilon_{0, \boldsymbol{k}}+c_{0} n_{0}
\end{array}\right)\left(\begin{array}{c}
\hat{a}_{0, \boldsymbol{k}} \\
\hat{a}_{0,-\boldsymbol{k}}^{\dagger}
\end{array}\right), \\
\hat{H}_{\text {polar }}^{(2)}=\sum_{\boldsymbol{k} \neq \mathbf{0}}\left(\hat{a}_{1, \boldsymbol{k}}^{\dagger}, \hat{a}_{-1,-\boldsymbol{k}}\right)\left(\begin{array}{cc}
\epsilon_{1, \boldsymbol{k}}+c_{2} n_{0} & c_{2} n_{0}-c_{f} n_{0} \\
c_{2} n_{0}-c_{f} n_{0} & \epsilon_{1, k}+c_{2} n_{0}
\end{array}\right)\left(\begin{array}{c}
\hat{a}_{1, \boldsymbol{k}} \\
\hat{a}_{-1,-\boldsymbol{k}}^{\dagger}
\end{array}\right) .
\end{gathered}
$$

Employing the Bogoliubov transformation, we diagonalize Eqs. (A10) and (A11) and then obtain the energy spectra:

$$
\begin{gathered}
E_{\mathrm{D}}^{(\mathrm{P})}(k)=\sqrt{\epsilon_{0, k}\left(\epsilon_{0, k}+2 c_{0} n_{0}\right)}, \\
E_{\mathrm{S}}^{(\mathrm{P})}(k)= \begin{cases}-\sqrt{\left(\epsilon_{0, \boldsymbol{k}}+q+c_{2} n_{0}\right)^{2}-\left(c_{2} n_{0}-c_{f} n_{0}\right)^{2}} & \left(q / c_{2} n_{0}<-1\right), \\
\sqrt{\left(\epsilon_{0, k}+q+c_{2} n_{0}\right)^{2}-\left(c_{2} n_{0}-c_{f} n_{0}\right)^{2}} & \text { (otherwise). }\end{cases}
\end{gathered}
$$

The emergence of the negative excitation energy in Eq. (A13) is attributed to negative values appearing in the diagonal matrix elements in Eq. (A11). We actually confirm that $\epsilon_{1, k}+c_{2} n_{0}$ can have negative values when $q / c_{2} n_{0}<-1$. This causes the inconsistency to determine the Bogoliubov coefficients if we choose the positive branch of the spectra. However, when we choose the negative branch, such problem does not occur in the region $q / c_{2} n_{0}<-1$.

\section{Antiferromagnetic state}

We consider the AFM state $\eta_{\mathrm{AFM}}=(1,0,1) / \sqrt{2}$ and derive the energy spectra. In a similar manner to the polar state, we obtain the Bogoliubov Hamiltonian:

$$
\hat{H}_{\mathrm{AFM}}=\hat{H}_{\mathrm{AFM}}^{(1)}+\hat{H}_{\mathrm{AFM}}^{(2)}+\text { const. }
$$




$$
\begin{gathered}
\hat{H}_{\mathrm{AFM}}^{(1)}=\frac{1}{2} \sum_{\boldsymbol{k} \neq \mathbf{0}}\left(\hat{a}_{0, \boldsymbol{k}}^{\dagger}, \hat{a}_{0,-\boldsymbol{k}}\right)\left(\begin{array}{cc}
\epsilon_{0, \boldsymbol{k}}-q+c_{2} n_{0} & c_{2} n_{0}-c_{f} n_{0} \\
c_{2} n_{0}-c_{f} n_{0} & \epsilon_{0, \boldsymbol{k}}-q+c_{2} n_{0}
\end{array}\right)\left(\begin{array}{c}
\hat{a}_{0, \boldsymbol{k}} \\
\hat{a}_{0,-\boldsymbol{k}}^{\dagger}
\end{array}\right), \\
\hat{H}_{\mathrm{AFM}}^{(2)}=\frac{1}{2} \sum_{\boldsymbol{k} \neq \mathbf{0}}\left(\hat{a}_{1, \boldsymbol{k}}^{\dagger}, \hat{a}_{-1, \boldsymbol{k}}^{\dagger}, \hat{a}_{1,-\boldsymbol{k}}, \hat{a}_{-1,-\boldsymbol{k}}\right)\left(\begin{array}{cccc}
\epsilon_{0, \boldsymbol{k}}+a & b & a & b \\
b & \epsilon_{0, \boldsymbol{k}}+a & b & a \\
a & b & \epsilon_{0, \boldsymbol{k}}+a & b \\
b & a & b & \epsilon_{0, \boldsymbol{k}}+a
\end{array}\right)\left(\begin{array}{c}
\hat{a}_{1, \boldsymbol{k}} \\
\hat{a}_{-1, \boldsymbol{k}} \\
\hat{a}_{1,-\boldsymbol{k}}^{\dagger} \\
\hat{a}_{-1,-k}^{\dagger}
\end{array}\right)
\end{gathered}
$$

with $a=\left(c_{0}+c_{2}\right) n_{0} / 2$ and $b=\left(c_{0}-c_{2}\right) n_{0} / 2$. Diagonalizing Eqs. (A15) and (A16) via the Bogoliubov transformation, we can obtain the following energy spectra:

$$
\begin{aligned}
& E_{\mathrm{D}}^{(\mathrm{AFM})}(k)=\sqrt{\epsilon_{0, \boldsymbol{k}}\left(\epsilon_{0, k}+2 c_{0} n_{0}\right)}, \\
& E_{\mathrm{S}_{\mathrm{z}}}^{(\mathrm{AFM})}(k)=\sqrt{\epsilon_{0, k}\left(\epsilon_{0, k}+2 c_{2} n_{0}\right)}, \\
& E_{\mathrm{S}_{\mathrm{xy}}}^{(\mathrm{AFM})}(k)= \begin{cases}-\sqrt{\left(\epsilon_{0, k}-q+c_{2} n_{0}\right)^{2}-\left(c_{2} n-c_{f} n_{0}\right)^{2}} & \left(q / c_{2} n_{0}>1\right), \\
\sqrt{\left(\epsilon_{0, k}-q+c_{2} n_{0}\right)^{2}-\left(c_{2} n-c_{f} n_{0}\right)^{2}} & \text { (otherwise). }\end{cases}
\end{aligned}
$$

As is the case with the polar state, the negative excitation energy emerges in Eq. (A19). The origin is negative values of the diagonal matrix elements in Eq. (A15).

\section{APPENDIX B: CRITICALITY OF THE PHASE TRANSITIONS}

In Sec. VII, we discuss the criticality of three phase transitions, namely BA-polar, BA-TBA, and FM-TBA transitions in Fig. 3(a). This section gives the detailed derivation of the critical exponents under the mean-field approximation.

\section{BA-polar transition}

It is convenient to introduce the dimensionless parameters $x=q /\left|c_{2}\right| n_{0}$ and $y=c_{f} /\left|c_{2}\right|$. Then, we can specify the parameters close to the BA-polar transition as shown in Fig. 7(a), which is given by

$$
\left(\begin{array}{l}
x \\
y
\end{array}\right)=\left(\begin{array}{c}
x_{0} \\
x_{0}-2
\end{array}\right)+r\left(\begin{array}{c}
\cos \theta \\
\sin \theta
\end{array}\right)
$$

with the parameter $x_{0} \in(1,2]$. Using this notation, we reexpress $\alpha$ and find that it is proportional to $r$ :

$$
\alpha=\frac{1}{2} \sqrt{\frac{r \sin \theta-r \cos \theta}{x_{0}+r \sin \theta}} \propto r^{1 / 2} \quad(r \ll 1) .
$$

Then, the transverse $F_{\perp}$ and the longitudinal $F_{z}$ amplitudes of the spin density vector in the BA state become

$$
\begin{aligned}
F_{\perp}(x, y) & =\sqrt{F_{x}^{2}+F_{y}^{2}} \\
& =2 n_{0} \alpha \sqrt{2-4 \alpha^{2}} \\
& \propto r^{1 / 2} \quad(r \ll 1), \\
F_{z}(x, y)=0 . &
\end{aligned}
$$

Here, we use the expression of $\eta_{\mathrm{BA}}$ in Eq. (18).

\section{BA-TBA transition}

We consider the BA-TBA phase transition in Fig. 7(b), in which the parameters close to the transitions are parametrized by

$$
\left(\begin{array}{l}
x \\
y
\end{array}\right)=\left(\begin{array}{c}
x_{0} \\
-x_{0}
\end{array}\right)+r\left(\begin{array}{c}
\cos \theta \\
\sin \theta
\end{array}\right)
$$

with the parameter $x_{0} \in(0,1)$. Then, by using Eq. (B5), $\alpha$ and $\beta$ are approximated to

$$
\begin{gathered}
\alpha \simeq \frac{1}{2} \sqrt{\frac{2-2 x_{0}}{2-x_{0}}}\left(1+\frac{1}{2} f\left(\theta, x_{0}\right) r\right) \quad(r \ll 1), \\
\beta \simeq g\left(\theta, x_{0}\right) r^{1 / 2} \quad(r \ll 1)
\end{gathered}
$$

with the real functions $f\left(\theta, x_{0}\right)$ and $g\left(\theta, x_{0}\right)$ defined by

$$
\begin{gathered}
f\left(\theta, x_{0}\right)=\frac{\sin \theta-\cos \theta}{2-2 x_{0}}-\frac{\sin \theta}{2-x_{0}}, \\
g\left(\theta, x_{0}\right)=\sqrt{-\frac{\sin \theta+\cos \theta}{x_{0}}} .
\end{gathered}
$$

Substituting Eqs. (B6) and (B7) into the expressions $F_{\perp}$ and $F_{z}$ for the TBA state, we can derive

$$
\begin{aligned}
F_{\perp}(x, y) & =2 \sqrt{2} n_{0} \alpha \sqrt{1-2 \alpha^{2}-\frac{1}{2} \beta^{2}} \\
& \Leftrightarrow F_{\perp}(x, y)-F_{\perp}\left(x_{0},-x_{0}\right) \propto r \quad(r \ll 1),
\end{aligned}
$$

$$
\begin{aligned}
F_{z}(x, y) & =-2 n_{0} \alpha \beta \\
& \simeq-\sqrt{\frac{2-2 x_{0}}{2-x_{0}}} n_{0} g\left(\theta, x_{0}\right) r^{1 / 2} \quad(r \ll 1) \\
& \propto r^{1 / 2} .
\end{aligned}
$$

\section{FM-TBA transition}

Finally, we calculate the critical exponents of the FM-TBA transition as shown in Fig. 7(c). The parameters close to the 
phase transition points are specified by

$$
\left(\begin{array}{l}
x \\
y
\end{array}\right)=\left(\begin{array}{c}
0 \\
y_{0}
\end{array}\right)+r\left(\begin{array}{c}
\cos \theta \\
\sin \theta
\end{array}\right)
$$

with the parameter $y_{0} \in(0,-2)$. Then, $\alpha$ and $\beta$ can be calculated as

$$
\begin{aligned}
& \alpha \simeq \frac{1}{2}\left(1-\frac{r \cos \theta}{4+2 y_{0}}\right) \quad(r \ll 1), \\
& \beta \simeq 1+\frac{r \cos \theta}{2 y_{0}} \quad(r \ll 1) .
\end{aligned}
$$

In a similar manner used in the other cases, we can derive

$$
\begin{aligned}
F_{\perp}(x, y) & =2 \sqrt{2} n_{0} \alpha \sqrt{1-2 \alpha^{2}-\frac{1}{2} \beta^{2}} \\
& \simeq n_{0} \sqrt{r \cos \theta} \sqrt{\frac{1}{2+y_{0}}-\frac{1}{y_{0}}} \quad(r \ll 1) \\
& \propto r^{1 / 2}, \\
F_{z}(x, y) & =-2 n_{0} \alpha \beta \\
& \simeq-n_{0}+n_{0} r \cos \theta\left(\frac{1}{4+2 y_{0}}-\frac{1}{2 y_{0}}\right) \quad(r \ll 1) \\
& \Leftrightarrow F_{z}(x, y)-F_{z}\left(0, y_{0}\right) \propto r .
\end{aligned}
$$

In the main text, we also mention the dynamical critical exponent $z$, which can be seen from Green's function about the transverse spin fluctuation. By applying the Bogoliubov theory to the FM state, it is obtained as

$$
G_{S}(\omega, \mathbf{k})=\frac{1}{-\hbar \omega+\frac{\hbar^{2} k^{2}}{2 M}-q} .
$$

This result means that $z=2$ and $q$ plays the same role with the chemical potential in a single-component Bose gas [69]. Since we treat three dimensions, which is larger than the upper critical dimension of a single-component Bose gas $(d=2)$, the mean-field level treatment above is reasonable.

\section{Comments on existence of the second-order phase transition}

Recently, Mathey and Diehl have discussed existence of second-order phase transitions in open Floquet systems [76] and unveiled absence of criticality in a stochastic model under a periodic driving forces by employing the perturbative renormalization group method. However, our system considered here does not include stochastic noises, and then their results cannot readily apply to our Floquet spinor Bose gas.

Also, we comment on higher-order corrections in the highfrequency expansion. Our results are based on the leading high-frequency expansion, and the condition $\left|c_{f} / c_{2}\right| \ll 1$ is necessary to assure the validity. Thus, the TP phase in Fig. 3(a) is clearly breaks the condition, so that we expect that the phase may be difficult to realize and the order of the transition may change. We leave the higher order effects on the Floquet spinor Bose gas including the realization of the TP phase and the criticality for other phases close to $\left|c_{f} / c_{2}\right|=1$ as a future work.
[1] M. Sigrist and K. Ueda, Rev. Mod. Phys. 63, 239 (1991).

[2] M. R. Norman, Science 332, 196 (2011).

[3] D. Vollhardt and P. Wölfle, The Superfluid Phases of Helium 3 (Taylor and Francis, London, 1990).

[4] G. E. Volovik, The Universe in a Helium Droplet (Oxford University Press, New York, 2003).

[5] R. Tamagaki, Prog. Theor. Phys. 44, 905 (1970).

[6] J. M. Lattimer and M. Prakash, Science 304, 536 (2004).

[7] M. Alford, K. Rajagopal, and F. Wilczek, Phys. Lett. B 422, 247 (1998).

[8] A. W. Steiner, S. Reddy, and M. Prakash, Phys. Rev. D 66, 094007 (2002).

[9] K. Kasamatsu, M. Tsubota, and M. Ueda, Int. J. Mod. Phys. B 19, 1835 (2005).

[10] Y. Kawaguchi and M. Ueda, Phys. Rep. 520, 253 (2012).

[11] D. M. Stamper-Kurn and M. Ueda, Rev. Mod. Phys. 85, 1191 (2013).

[12] V. I. Yukalov, Laser Phys. 28, 053001 (2018).

[13] T. Lahaye, C. Menotti, L. Santos, M. Lewenstein, and T. Pfau, Rep. Prog. Phys. 72, 126401 (2009).

[14] M. Baranov, M. Dalmonte, G. Pupillo, and P. Zoller, Chem. Rev. 112, 5012 (2012).

[15] J. Stenger, S. Inouye, D. M. Stamper-Kurn, H.-J. Miesner, A. P. Chikkatur, and W. Ketterle, Nature (London) 396, 345 (1998).

[16] E. M. Bookjans, A. Vinit, and C. Raman, Phys. Rev. Lett. 107, 195306 (2011).
[17] D. Jacob, L. Shao, V. Corre, T. Zibold, L. De Sarlo, E. Mimoun, J. Dalibard, and F. Gerbier, Phys. Rev. A 86, 061601(R) (2012).

[18] C. Becker, S. Stellmer, P. Soltan-Panahi, S. Dörscher, M. Baumert, E.-M. Richter, J. Kronjäger, K. Bongs, and K. Sengstock, Nat. Phys. 4, 496 (2008).

[19] D. Yan, J. J. Chang, C. Hamner, P. G. Kevrekidis, P. Engels, V. Achilleos, D. J. Frantzeskakis, R. Carretero-González, and P. Schmelcher, Phys. Rev. A 84, 053630 (2011).

[20] C. Hamner, J. J. Chang, P. Engels, and M. A. Hoefer, Phys. Rev. Lett. 106, 065302 (2011).

[21] C. Hamner, Y. Zhang, J. J. Chang, C. Zhang, and P. Engels, Phys. Rev. Lett. 111, 264101 (2013).

[22] T. M. Bersano, V. Gokhroo, M. A. Khamehchi, J. D. Ambroise, D. J. Frantzeskakis, P. Engels, and P. G. Kevrekidis, Phys. Rev. Lett. 120, 063202 (2018).

[23] L. S. Leslie, A. Hansen, K. C. Wright, B. M. Deutsch, and N. P. Bigelow, Phys. Rev. Lett. 103, 250401 (2009).

[24] J. Y. Choi, W. J. Kwon, and Y. I. Shin, Phys. Rev. Lett. 108, 035301 (2012).

[25] J. Y. Choi, S. Kang, S. W. Seo, W. J. Kwon, and Y. I. Shin, Phys. Rev. Lett. 111, 245301 (2013).

[26] D. S. Hall, M. W. Ray, K. Tiurev, E. Ruokokoski, A. H. Gheorghe, and M. Mättänen, Nat. Phys. 12, 478 (2016).

[27] W. Lee, A. H. Gheorghe, K. Tiurev, T. Ollikainen, M. Mättänen, and D. S. Hall, Sci. Adv. 4, eaao3820 (2018). 
[28] A. E. Leanhardt, Y. Shin, D. Kielpinski, D. E. Pritchard, and W. Ketterle, Phys. Rev. Lett. 90, 140403 (2003).

[29] S. W. Seo, W. J. Kwon, S. Kang, and Y. Shin, Phys. Rev. Lett. 116, 185301 (2016).

[30] E. Nicklas, M. Karl, M. Höfer, A. Johnson, W. Muessel, H. Strobel, J. Tomkovič, T. Gasenzer, and M. K. Oberthaler, Phys. Rev. Lett. 115, 245301 (2015).

[31] M. Anquez, B. A. Robbins, H. M. Bharath, M. Boguslawski, T. M. Hoang, and M. S. Chapman, Phys. Rev. Lett. 116, 155301 (2016).

[32] M. Prüfer, P. Kunkel, H. Strobel, S. Lannig, D. Linnemann, C.-M. Schmied, J. Berges, T. Gasenzer, and M. K. Oberthaler, Nature (London) 563, 217 (2018).

[33] I. Bloch, J. Dalibard, and W. Zwerger, Rev. Mod. Phys. 80, 885 (2008).

[34] M. Bukov, L. D’Alessio, and A. Polkovnikov, Adv. Phys. 64, 139 (2015).

[35] A. Eckardt, Rev. Mod. Phys. 89, 011004 (2017).

[36] P. Weinberg, M. Bukov, L. D’Alessio, A. Polkovnikov, S. Vajna, and M. Kolodrubetz, Phys. Rep. 688, 1 (2017).

[37] T. Oka and S. Kitamura, Annu. Rev. Condens. Matter Phys. 10, 387 (2019).

[38] T. Oka and H. Aoki, Phys. Rev. B 79, 081406(R) (2009).

[39] A. Zenesini, H. Lignier, C. Sias, O. Morsch, D. Ciampini, and E. Arimondo, Laser Phys. 20, 1182 (2010).

[40] J. Struck, C. Ölschläger, R. Le Targat, P. Soltan-Panahi, A. Eckardt, M. Lewenstein, P. Windpassinger, and K. Sengstock, Science 333, 996 (2011).

[41] M. Aidelsburger, M. Atala, M. Lohse, J. T. Barreiro, B. Paredes, and I. Bloch, Phys. Rev. Lett. 111, 185301 (2013).

[42] H. Miyake, G. A. Siviloglou, C. J. Kennedy, W. C. Burton, and W. Ketterle, Phys. Rev. Lett. 111, 185302 (2013).

[43] G. Jotzu, M. Messer, R. Desbuquois, M. Lebrat, T. Uehlinger, D. Greif, and T. Esslinger, Nature (London) 515, 237 (2014).

[44] N. Fläschner, D. Vogel, M. Tarnowski, B. S. Rem, D.-S. Lühmann, M. Heyl, J. C. Budich, L. Mathey, K. Sengstock, and C. Weitenberg, Nat. Phys. 14, 265 (2018).

[45] N. Goldman, G. Juzeliūnas, P. Öhberg, and B. Spielman, Rep. Prog. Phys. 77, 126401 (2014).

[46] N. Goldman and J. Dalibard, Phys. Rev. X 4, 031027 (2014).

[47] V. Galitski, G. Juzeliūnas, and I. B. Spielman, Phys. Today 72(1), 38 (2019).

[48] V. Galitski and I. B. Spielman, Nature (London) 494, 49 (2013).

[49] F. Gerbier, A. Widera, S. Folling, O. Mandel, and I. Bloch, Phys. Rev. A 73, 041602(R) (2006).

[50] S. R. Leslie, J. Guzman, M. Vengalattore, J. D. Sau, M. L. Cohen, and D. M. Stamper-Kurn, Phys. Rev. A 79, 043631 (2009).
[51] J. Guzman, G.-B. Jo, A. N. Wenz, K. W. Murch, C. K. Thomas, and D. M. Stamper-Kurn, Phys. Rev. A 84, 063625 (2011).

[52] A. Tokuno and S. Uchino, Phys. Rev. A 87, 061604(R) (2013).

[53] A. Eckardt and E. Anisimovas, New J. Phys. 17, 093039 (2015).

[54] T. Mikami, S. Kitamura, K. Yasuda, N. Tsuji, T. Oka, and H. Aoki, Phys. Rev. B 93, 144307 (2016).

[55] R. A. Williams, L. J. LeBlanc, K. Jiménez-García, M. C. Beeler, A. R. Perry, W. D. Phillips, and I. B. Spielman, Science 335, 314 (2012).

[56] L. Pitaevskii and S. Stringari, Bose-Einstein Condensation, International Series of Monographs on Physis (Oxford Science Publications, Oxford, UK, 2003).

[57] There can exist metastable states in presence of the linear Zeeman effect breaking $Z_{2}$ symmetry [58-61].

[58] H.-J. Miesner, D. M. Stamper-Kurn, J. Stenger, S. Inouye, A. P. Chikkatur, and W. Ketterle, Phys. Rev. Lett. 82, 2228 (1999).

[59] E. Witkowska, J. Dziarmaga, T. Świsłocki, and M. Matuszewski, Phys. Rev. B 88, 054508 (2013).

[60] T. Świsłocki, A. Zembrzuski, M. Matuszewski, and E. Witkowska, Phys. Rev. A 97, 033629 (2018).

[61] J. H. Kim, D. H. Hong, S. Kang, and Y. Shin, Phys. Rev. A 99, 023606 (2019).

[62] Y. Shin (private communication).

[63] K. Murata, H. Saito, and M. Ueda, Phys. Rev. A 75, 013607 (2007).

[64] N. T. Phuc, Y. Kawaguchi, and M. Ueda, Phys. Rev. A 88, 043629 (2013).

[65] V. Novicenko, E. Anisimovas, and G. Juzeliunas, Phys. Rev. A 95, 023615 (2017).

[66] B. Evrard, A. Qu, K. Jimenez-Garcia, J. Dalibard, and F. Gerbier, Phys. Rev. A 100, 023604 (2019).

[67] R. Barnett, A. Turner, and E. Demler, Phys. Rev. Lett. 97, 180412 (2006).

[68] A. Lamacraft, Phys. Rev. Lett. 98, 160404 (2007).

[69] S. Sachdev, Quantum Phase Transitions (Cambridge University Press, Cambridge, UK, 2011).

[70] T. Mori, T. Kuwahara, and K. Saito, Phys. Rev. Lett. 116, 120401 (2016).

[71] T. Kuwahara, T. Mori, and K. Saito, Ann. Phys. 367, 96 (2016).

[72] D. A. Abanin, W. De Roeck, W. W. Ho, and F. Huveneers, Phys. Rev. B 95, 014112 (2017).

[73] D. Abanin, W. De Roeck, F. Huveneers, and W. W. Ho, Commun. Math. Phys. 354, 809 (2017).

[74] M.-S. Chang, C. D. Hamley, M. D. Barrett, J. A. Sauer, K. M. Fortier, W. Zhang, L. You, and M. S. Chapman, Phys. Rev. Lett. 92, 140403 (2004)

[75] H. Saito and M. Ueda, Phys. Rev. A 72, 053628 (2005).

[76] S. Mathey and S. Diehl, Phys. Rev. Lett. 122, 110602 (2019). 